\title{
Tuberculosis extrapulmonar como apendicitis aguda tuberculosa
}

\section{Extrapulmonary tuberculosis as acute tuberculous apendicitis}

\author{
Jairo Jaird Vergara-Corena, luis Alfredo Blanco-Castiblanco, \\ Gilberto Rafael Mangones-Ortega, Keimer José Mejía-Flórez • Sincelejo (Colombia)
}

DOI: https://doi.org/10.36104/amc.2021.1896

\section{Resumen}

Introducción: la apendicitis es la emergencia quirúrgica abdominal más frecuente, pero la tuberculosis como agente etiológico hace especial este caso, debido a su muy baja frecuencia entre 0.1 y $0.6 \%$, su hallazgo incidental nos orienta a buscar otros órganos comprometidos y a iniciar tratamiento específico, este es el primer reporte de caso en nuestra región.

Reporte de caso: mujer de 33 años, con antecedentes de insuficiencia suprarrenal primaria, en tratamiento con fludrocortisona, quien consultó por presentar dolor en cuadrante inferior derecho abdominal, picos febriles y episodios eméticos, lo anterior asociado a biometría hemática con leucocitosis más desviación a la izquierda, uroanálisis patológico, se sospecha infección del tracto urinario, iniciando antibioticoterapia sin obtener mejoría, por lo que se evalúa por cirugía general que considera abdomen agudo de origen en apendicitis, realizando abordaje quirúrgico con posterior estudio histopatológico que sugiere inflamación granulomatosa caseificante compatible con tuberculosis, con la consecuente demostración de BAAR mediante tinción de Ziehl Neelsen.

Conclusión: la tuberculosis extrapulmonar tiene presentaciones clínicas diversas, por lo tanto, debemos mantener sospecha ante manifestaciones atípicas de la patología, la apendicitis aguda tuberculosa, generalmente se da en el contexto de un paciente inmunosuprimido y su diagnóstico etiológico es realizado en el posoperatorio, por lo tanto, es indispensable vigilar y revisar el resultado de la biopsia y las tinciones que nos permitan realizar tratamientos específicos. (Acta Med Colomb 2021; 46. DOI: https://doi.org/10.36104/amc.2021.1896).

Palabras clave: apendicitis, tuberculosis intestinal, abdomen agudo, tuberculosis extrapulmonar.

\begin{abstract}
Introduction: appendicitis is the most frequent abdominal surgical emergency, but tuberculosis as the etiological agent makes this case special, due to its very low frequency of 0.1 to $0.6 \%$. Its incidental finding guided us to seek other involved organs and begin specific treatment. This is the first case report in our region.

Case report: a 33-year-old woman with a history of primary adrenal insufficiency being treated with fludrocortisone consulted due to right lower quadrant abdominal pain, fever and emesis, associated with leukocytosis with a left shift and a pathological urinalysis. A urinary tract infection was suspected, and antibiotic therapy was begun with no improvement. She was therefore seen by general surgery, who suspected acute abdomen arising from appendicitis. She underwent surgery with a subsequent histopathological study which suggested caseifying granulomatous inflammation compatible with tuberculosis, with ensuing proof of acid-fast bacilli using Ziehl Neelsen staining.

Conclusion: Extrapulmonary tuberculosis has diverse clinical presentations. Therefore, when faced with atypical manifestations of this disease we should maintain a level of suspicion. Acute tuberculous appendicitis generally presents in the context of an immunosuppressed patient, and its etiological diagnosis is carried out after surgery. Therefore, it is essential to be vigilant and review the biopsy result and stains, which will allow specific treatments to be carried out. (Acta Med Colomb 2021; 46. DOI: https://doi.org/10.36104/amc.2021.1896).
\end{abstract}

Key words: appendicitis, intestinal tuberculosis, acute abdomen, extrapulmonary tuberculosis.
Dr. Jairo Jaird Vergara-Corena: Médico Internista Universidad de Sucre, Clínica Salud Social; Dr. Luis Alfredo Blanco-Castiblanco: Médico Urgencias; Dr. Gilberto Rafael MangonesOrtega: Cirugía General y Laparoscópica; Dr. Keimer José Mejía-Flórez: Médico Urgencias. Clínica Salud Social. Sincelejo (Colombia). Correspondencia: Dr. Jairo Jaird VergaraCorena. Sincelejo (Colombia). E- mail: jairovergara2011@gmail.com Recibido: 27/V/2020 Aceptado: 22/VII/2020 


\section{Introducción}

La apendicitis es la emergencia quirúrgica abdominal más frecuente, se inicia por obstrucción del apéndice por hiperplasia linfoide, parasitosis, cuerpos extraños, neoplasias, entre otros. Se estima que su origen en tuberculosis varía entre 0.1 y $0.6 \%(1,2)$, convirtiéndose en una condición infrecuente y de dificultad diagnostica, lo que vuelve muy interesante esta forma de presentación clínica de este caso.

La tuberculosis (TB) es una enfermedad infectocontagiosa, con agente causal en el bacilo de Koch, una bacteria aerobia estricta, ácido alcohol resistente, capaz de generar cambios fibróticos e inflamatorios de predominio pulmonar. Las formas extrapulmonares constituyen el 10-30\% de los casos, siendo la TB del tubo digestivo responsable del $1 \%$ de dichas formas $(2,3)$.

\section{Reporte de caso}

Mujer de 33 años con antecedentes de insuficiencia suprarrenal primaria, en tratamiento con fludrocortisona, quien consultó por presentar dolor abdominal en flanco y fosa iliaca derecha, asociado a picos febriles y episodios eméticos, ingresando álgida, con abdomen doloroso a la palpación generalizada, aunque sin signos de irritación peritoneal, indicándose biometría hemática que revelaba leucocitosis a expensas de neutrófilos y uroanálisis con leucocituria y bacteriuria, se consideró una posible infección del tracto urinario y se indicó antibioticoterapia empírica con una cefalosporina de tercera generación, previo urocultivo y se solicita ecografía abdominal total, la cual es normal, luego de 24 horas de manejo cursa sin mejoría clínica, por lo cual es evaluada por el servicio de cirugía general, que considera apendicitis aguda probable. Se realiza apendicectomía por abordaje de Rocky Davis, identificando apéndice cecal fibrinopurulenta (Figura 1), con peritonitis localizada y con consecuente examen histopatológico que reporta inflamación crónica granulomatosa caseificante compatible con infección por TB, indicándose tinción de Ziehl Neelsen, la cual es positiva para BAAR (Figuras 2, 3).

Tras la confirmación microbiológica se inicia fase inicial (dos meses) de tratamiento antituberculoso con rifampicina, isoniacida, pirazinamida y etambutol (RHZE), con el consecuente seguimiento al régimen de tratamiento por salud pública y realización de estudios imagenológicos contrastados, los cuales a pesar de ser asintomática respiratoria revela cambios parenquimatosos pulmonares a favor de patrón miliar y engrosamiento periomental en relación a cambios histológicos descritos.

\section{Discusión}

La TB es un problema de salud pública, el cual ha venido en aumento debido a las malas condiciones higiénico-sanitarias, la migración, inmunodeficiencias y uso de la terapia con inmunosupresores (4). Para Colombia, se estimaba en 2011 una incidencia de 24.5/100 000 habitantes, con un aumento de la enfermedad extrapulmonar de 14-20\% (5).

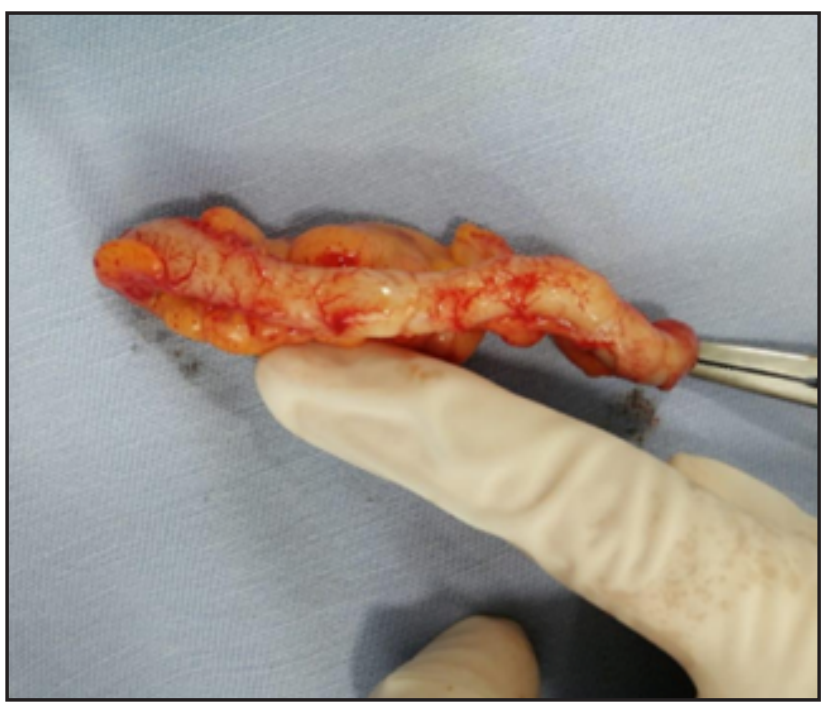

Figura 1: Apéndice cecal fibrinopurulenta, que mide $7 * 1.5 \mathrm{~cm}$.

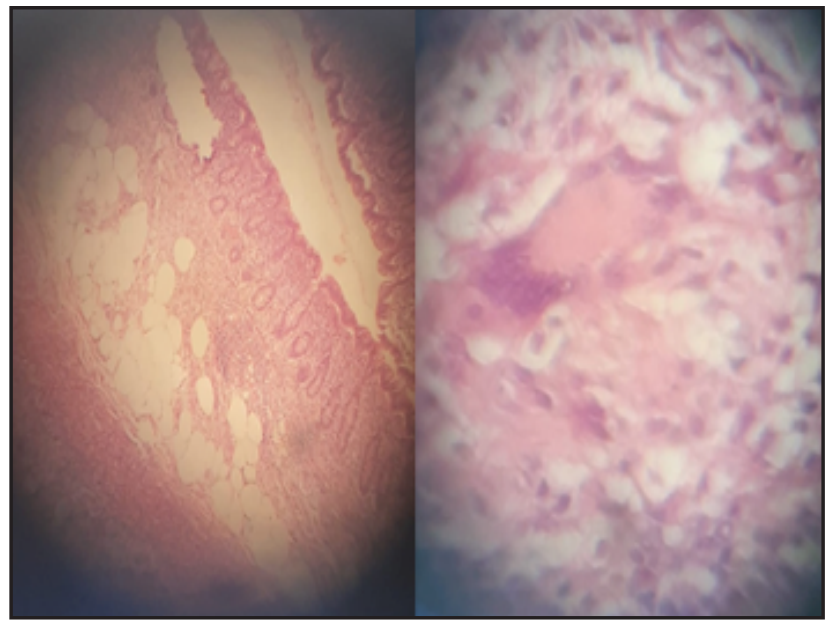

Figura 2. Apéndice cecal proceso inflamatorio crónico.

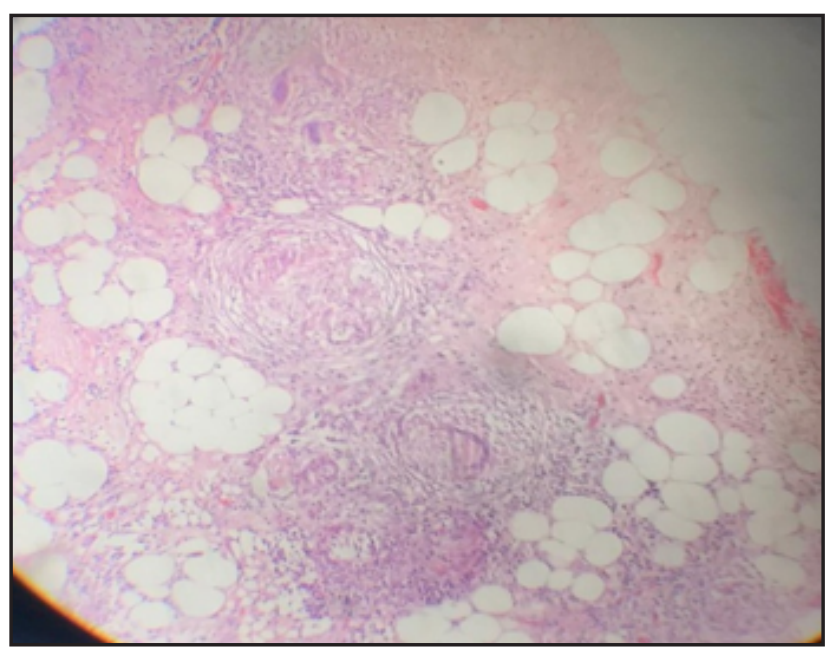

Figura 3. Epiplón, tejido adiposo con presencia de células granulomatoso, con presencia de células gigantes multinucleadas tipo langhans y necrosis caseosa rodeada de linfocitos. 
En la integración clínica retrospectiva de este caso, es importante destacar las condiciones de salud preexistentes, el uso de medicamentos inmunosupresores y su relación con la manifestación clínica principal que fue el dolor abdominal, los diagnósticos diferenciales de infección urinaria vs manifestaciones urinarias de una apendicitis aguda, que ya hay descritos uroanálisis patológico con leucocituria, hematuria y síntomas irritativos urinarios en apendicitis aguda (6), nos pueden llevar a cometer errores de diagnóstico sindromático de ingreso en los pacientes y no es extraño que ocurra, esta es una enseñanza del caso, es muy difícil pensar en TB apendicular como etiología de la apendicitis, primero porque no es la primera ubicación de TB extrapulmonar y la localización abdominal de la TB es menos frecuente que otras localizaciones extrapulmonares, como son la linfática, genitourinaria, ósea y meníngea (7). La afectación abdominal radica en $75 \%$ de los casos en zonas con mayor presencia de tejido linfoide y gran capacidad de absorción como son la cavidad peritoneal, la región ileocecal y yeyuno-ileal, así como en sus ganglios de drenaje mesentérico y paraaórticos superiores (8).

Los métodos diagnósticos que utilizamos fueron acertados y la literatura nos apoya, es necesario el aislamiento e identificación microbiológica del mycobacterium tuberculosis. Los métodos con mayor sensibilidad y especificidad son el cultivo, la reacción en cadena de polimerasa y la tinción $(\mathrm{ZN})$; siendo esta ultima la más frecuentemente utilizada dado su bajo costo, con resultados oportunos para el inicio de un esquema antifímico (9).

Este caso es muy importante primero por lo atípico del cuadro clínico en su relación con el diagnóstico etiológico final, donde es poco probable el nivel de sospecha inicial de la patología, se hace fundamental el seguimiento en el posoperatorio y la revisión de los métodos diagnósticos en este caso biopsia, tinciones especiales, se convierte en el primer reporte de caso en nuestra región, en el departamento de Sucre, aportando evidencia epidemiológica a los casos de tuberculosis extrapulmonar en Colombia y en la región caribe.

En cuanto al tratamiento, se indican tuberculostáticos de primera línea, (RHZE) durante seis meses en la mayoría de los casos, distribuidos en una fase inicial de dos meses y una fase de consolidación los posteriores cuatro meses $(10,11)$.

\section{Agradecimientos}

Agredecemos a la Clínica Salud Social y a la paciente, por ayudarnos al desarrollo de este tipo de evidencia científica, que permita aportar a la investigación en nuestro ámbito local.

\section{Referencias}

1. Sharath Chandra BJ, Girish TU, Thrishuli PB. Vinay HG. Primary Tuberculosis of the Appendix: A Rare Cause of a Common Disease. J Surg Tech Case Rep. 2013;5(1):32-34.

2. Santana-Ortiz R, Ortiz-Mojica HG, Rodríguez-Rodríguez IC, BaltazarGuerrero JE, Pérez-Rulfo ID, Duque-Zepeda F, Orozco-Pérez J, YanowskyReyes G. Apendicitis tuberculosa, Rev Med MD 2018; 9.10 (3)

3. Montiel-Jarquín AJ, Alvarado-Ortega I, Romero-Figueroa MS, RodríguezPérez F, Rodríguez-Lima F, Loria-Castellanos J. Abdomen agudo ocasionado por apendicitis aguda de probable origen tuberculoso. Informe de un caso. Iatreia. 2017 Jul-Sept;30(3): 321-325. DOI 10.17533/udea.iatreia.v30n3a07

4. Guirat A, Koubaa M, Mzali R, Abid B,Ellouz S, Affes, et al. Peritoneal tuberculosis. Clin Res Hepatol Gastroenterol., 35 (2011), pp. 60-69

5. Solier Insuasty J, Bolivar A, Calvo LS ,Roberto SL . Tuberculosis peritoneal simulando cáncer de ovario. Peritoneal tuberculosis simulating ovarian cancer. Acta Médica Colomb., 2008 (2014), pp. 383-387

6. Tundidor A, Amado J, Montes J. manifestaciones urinarias de la apendicitis aguda. Arch. Esp. Urol. 2005; 58: 207-212.

7. Marshall JB. Tuberculosis of the gastrointestinal tract and peritoneum. Am J Gastroenterol 1993;88:899-999

8. Rodríguez E, Beltrán E , Ribas J , Torres M. apendicitis aguda como inicio de una tuberculosis generalizada. Gastroenterol Hepatol 2004;27(1):43-4

9. Gan HT, Chen YQ, Ouayang Q, bu H, Yang XY. Differentiation between intestinal tuberculosis and Chron's disease in endoscopic biopsy specimens by polymerase chain reaction. Am J Gastroenterol 2002; 97: 1446-1451

10. Saigal S, Agarwal SR, Nandeesh HP, Sarin SK . Safety of an ofloxacin-based antitubercular regimen for the treatment of tuberculosis in patients with underlying chronic liver disease: A preliminary report. J Gastroenterol Hepatol., 16 (2001), pp. 1028-1032

11. Udwadia ZF, Sen T. Pleural tuberculosis: an update. Curr Opin Pulm Med. 2010 Jul;16(4):399-406 